\title{
Descrição do padrão alimentar e da saúde bucal de crianças do sul do Brasil
}

\section{Description of dietary patterns and oral health condition of children from a city in southern of Brazil}

\author{
Márcia Cançado Figueiredo* \\ Claudia Bernardete Sena Ribeiro ${ }^{* *}$ \\ Marina Silveira Noronha** \\ Kátia Valença Correia Leandro da Silva ${ }^{* * * *}$
}

\section{Resumo}

Introdução: para proporcionar ações efetivas na busca pela saúde bucal, o acesso à saúde deve ser singularizado conforme as necessidades da população e, isso, deve ser planejado a partir de levantamentos e estudos que evidenciem o perfil da comunidade, servindo de parâmetro para o planejamento de futuras ações. Objetivos: descrever as condições de saúde bucal e o padrão alimentar de crianças e correlacioná-las com o perfil socioeconômico de suas famílias cadastradas na Estratégia Saúde da Família Augusta Meneguine do município de Viamão-RS. Metodologia: trata-se de um estudo longitudinal observacional, em que foram coletados dados de 212 crianças, de zero a 12 anos de idade e de suas famílias durante visitas domiciliares. Resultados: $42 \%$ das pessoas viviam com até um salário mínimo e $48,6 \%$ residiam em grupos de três a cinco pessoas por casa. Houve associação significante entre renda familiar de até um salário mínimo e mais de três ingestas de açúcar entre as refeições, de três a cinco salários mínimos e duas ingestas de açúcar entre as refeições; tipo de casa (alvenaria) e de dois a três vezes higiene bucal por dia $(p<0,05)$. As crianças apresentaram mais de $60 \%$ de dentes cariados, $10 \%$ de dentes perdidos e $40 \%$ de dentes obturados na faixa de sete a 12 anos de idade. Conclusão: por meio deste levantamento foi possível identificar um perfil carente de saúde bucal e de nível socioeconômico da população estudada, isto é, baixa remuneração e escolaridade dos cuidadores das crianças, altos índices de placa visível, sangramento gengival, cárie e perdas dentárias das crianças.

Palavras-chave: Crianças. Epidemiologia. Padrões alimentares. Saúde bucal.

\section{Introdução}

Segundo Wiest et al. ${ }^{1}$ (2008), muito mais que um ato biológico, a alimentação humana é um ato social e cultural. Para Filho e Batis$\operatorname{ta}^{2}$ (2008), a alimentação e seu reflexo biológico (a nutrição) representam os traços de relações mais fortes da vida humana com o ambiente. É por meio dos alimentos que se estabelece o ciclo de trocas com o meio físico, biótico e social, constituindo ecossistemas saudáveis ou insalubres.

Atualmente, vários estudos interdisciplinares têm identificado que a refeição em família contribui para o bom estado nutricional, relacional e para uma melhor qualidade de vida, principalmente entre os jovens. A pirâmide alimentar, criada pelo United States Department of Agriculture (Usda) ${ }^{3}$ sofreu diversas mudanças até chegar ao padrão atual (Figura 1). Além de uma divisão feita como referencial para o exercício físico e sua importância, essa informa o quanto de cada alimento é necessário aproximadamente ser consumido diariamente para se ter uma alimentação saudável:

Professora associada IV da Universidade Federal do Rio Grande do Sul (UFRGS), Porto Alegre, RS, Brasil; Mestra e Doutora em Odontopediatria pela Faculdade de Odontologia de Bauru, Universidade de São Paulo(USP), São Paulo, SP, Brasil

Graduanda da Faculdade de Odontologia da Universidade Federal do Rio Grande do Sul (UFRGS), Porto Alegre, RS, Brasil.

Graduanda da Faculdade de Odontologia da Universidade Federal do Rio Grande do Sul (UFRGS), Porto Alegre, RS, Brasil.

**** Grofessora associada IV da Universidade Federal do Rio Grande do Sul (UFRGS); Doutora em Genética e Biologia Molecular pela Universidade Federal do Rio Grande do Sul (UFRGS), Porto Alegre, RS, Brasil. 
- grupo dos pães, dos cereais, do arroz e das massas: 6 a 11 porções;

- grupo das verduras e dos legumes: 3 a 5 porções;

- grupo das frutas: 2 a 4 porções;

- grupo do leite, do iogurte, dos queijos: 2 a 3 porções;

- grupo das carnes, das aves, dos peixes, das leguminosas, dos ovos e das nozes: 2 a 3 porções;

- grupo das gorduras, dos óleos e dos açúcares: recomendação de uso moderado.

No entanto, é necessário perceber o que são essas porções citadas no guia alimentar. Uma porção do grupo grãos é uma fatia de pão, ou meia xícara de arroz ou de massa. Já uma porção de leite é um copo de leite, ou um copo de iogurte, ou ainda duas fatias de queijo. As porções variam entre os grupos ${ }^{4}$.

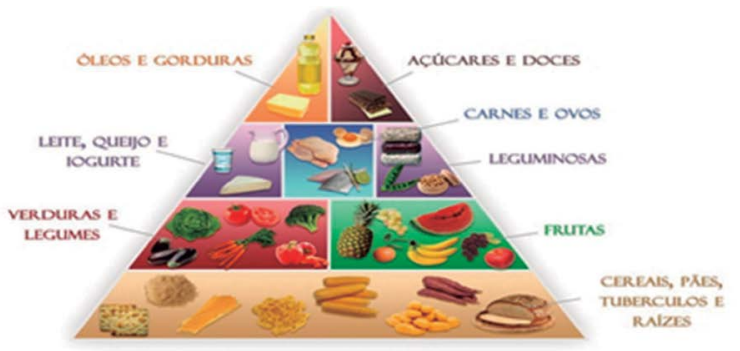

Fonte: dos autores.

Figura 1 - Modelo de pirâmide alimentar sugerida pelo Usda

Outra necessidade é adaptar esse guia nutricional americano aos padrões brasileiros de alimentação. Por serem alimentos comuns à dieta e de fácil acesso à população brasileira, as porções de frutas e vegetais podem ser aumentadas para três ou cinco porções no grupo das frutas e para quatro ou cinco porções no grupo dos vegetais. Em relação às leguminosas, principalmente o feijão, achou-se conveniente colocá-las a parte, uma vez que não têm os mesmos valores nutritivos que carnes e ovos e são os produtos isolados que mais contribuem para o consumo de proteínas, não podendo ser substituídas uma pela outra, sem o necessário ajuste no equilíbrio de aminoácidos, que é dado pelo consumo simultâneo desse alimento com o arroz ${ }^{5}$.

De acordo Batista et al. ${ }^{6}$ (2007), há certa concordância que o estado nutricional está inter-relacionado com a condição bucal, porém, há poucos dados disponibilizados na literatura para confirmar essa afirmação e para estabelecer qual seria a natureza de qualquer tipo de relação possível. Uma alimentação balanceada capaz de proporcionar um adequado estado nutricional, certamente, contribui para uma desejável condição bucal do indivíduo. Dessa forma, em casos de carências nutricionais ou mesmo a ingestão de alguns componentes alimentares específicos pode influenciar os processos de formação dental, erupção e desenvolvimento da cárie dentária ${ }^{7}$. Já a American Dietetic Association ${ }^{8}$ orienta que a nutrição é componente fundamen- tal para a saúde bucal e que tanto a alimentação como a nutrição tem influência direta na progressão da cárie dental. Doença essa, que ainda é uma das agressões mais prevalentes à saúde no mundo, constituindo-se um problema de saúde pública em nível mundial.

Há muito tempo, são conhecidos os fatores biológicos relacionados ao processo de desenvolvimento das doenças bucais como a cárie dentária, entretanto, cada vez mais, são adicionados aos determinantes desse processo fatores sociais, psicológicos e ambientais. Elementos como o desenvolvimento econômico e social, o nível educacional, os costumes e as tradições, o acesso aos serviços odontológicos e o ambiente físico em que os indivíduos estão inseridos, bem como seus hábitos de higiene e de alimentação são fatores que estão intimamente ligados ao percurso das enfermidades bucais, influenciando também no ritmo de sua expansão ${ }^{9}$.

É fato que as condições de saúde bucal, no Brasil e no mundo, melhoraram nas últimas décadas a partir das décadas de 1960 e 1970; porém, a cárie dentária, um dos principais males que acomete a cavidade bucal, permanece como um grande problema de saúde pública do país. De acordo com a pesquisa de Saúde Bucal no Brasil de $2010^{\mathbf{1 0}}$, uma criança brasileira aos 5 anos de idade tem, em média, 2,43 dentes com alguma experiência de cárie. Esses dados naturalmente variam bastante, sendo um dos motivos para essa grande diferença a marca da desigualdade social e salarial, que chama a atenção no cenário brasileiro, no qual essas desigualdades não estão presentes apenas entre as grandes regiões do país, mas também no interior das diversas regiões de um estado, e, até mesmo, nos diversos bairros de um município. A correlação entre renda familiar e CPO-D médio aos 12 anos destaca o impacto dessa grande variedade: cidades com renda média mais elevada tendem a apresentar menores médias de $\mathrm{CPO}^{11}$. No Brasil, estudo recente mostrou que o consumo de açúcar e refrigerantes participa/atua com $13,7 \%$ da energia consumida pelos indivíduos, caracterizando o aumento no consumo, já o excessivo consumo de açúcar refinado e refrigerante, como traço marcante e negativo da evolução do padrão alimentar brasileiro entre 1988 e 1996. Salienta-se que esse valor está acima do que a Organização Mundial da Saúde (OMS) recomenda como limite populacional máximo para o consumo de açúcar, isto é, $10 \%$ do consumo energético total ${ }^{12}$.

Há, por isso, que se considerarem os aspectos dietético-nutricionais novamente, como fatores culturais que contribuem de modo importante para a manutenção de hábitos alimentares deletérios e a consequente instalação de doenças bucais. Além da renda familiar, a inserção social tem influência direta na prevalência da cárie dentária ${ }^{13}$. Indivíduos com grau de escolaridade maior tendem a escovar seus dentes mais vezes, e suas crianças começam a ter seus dentes higienizados mais precocemente, 
com impacto significativo na saúde bucal. Somado a isso, a disponibilidade de instalações sanitárias adequadas também favorece as boas práticas de higiene pessoal ${ }^{14}$.

Paralelamente, apesar da queda das cifras de desnutrição em todo o mundo, concomitante com o aumento da inatividade física e o estresse do modo moderno de vida, que leva ao aumento da adiposidade corporal, existe o outro lado da história, que são os processos crônicos não transmissíveis, como o diabetes mellitus, a obesidade, a hipertensão arterial sistêmica, os processos degenerativos do sistema nervoso central, agrupando-se em comorbidades associadas à alimentação hipercalórica, às gorduras trans, aos ácidos graxos saturados, ao consumo excessivo do açúcar e do sal, às calorias vazias dos refrigerantes, ao uso imoderado do álcool, do fumo, ao sedentarismo e a outras práticas não saudáveis do estilo de vida ocidental ${ }^{2}$.

A OMS recomenda que estudos epidemiológicos deveriam ser realizados a cada cinco anos, dando uma noção da importância de se buscar a recuperação da saúde da população, usando métodos preventivos ou curativos, porém com ações baseadas em dados epidemiológicos locais, os quais irão permitir a identificação dos problemas mais prevalentes naquela comunidade para um adequado acompanhamento das condições de saúde bucal, bem como para avaliar as ações desenvolvidas, assim como, também sugeriu algumas idades-índices de 5 e 12 anos para serem usadas nos levantamentos para crianças $^{\mathbf{1 5}}$.

Diante do acima exposto, propõe-se, neste estudo, realizar uma avaliação do padrão alimentar, correlacionando-o com o perfil socioeconômico e a saúde bucal de crianças de 0 a 12 anos residentes no bairro Augusta Meneghini em Viamão-RS.

\section{Material e métodos}

O presente estudo é do tipo transversal, observacional e analítico. A amostra foi composta de 212 crianças de 0 a 12 anos de idade, cadastradas na Estratégia Saúde da Família da UBS Augusta Meneguine do município de Viamão-RS.

Os dados foram coletados por meio de visitas domiciliares realizadas na vila, aos sábados, nas quais, os responsáveis pelas crianças moradoras das casas visitadas responderam a um questionário padronizado, que buscava informações referentes às condições socioeconômicas do chefe da família, além de dados sobre a saúde geral, o padrão alimentar, o peso, a altura e a pressão arterial de todos os integrantes das 50 famílias visitadas, dados esses avaliados pela equipe da enfermagem e da nutrição (Figura 2).

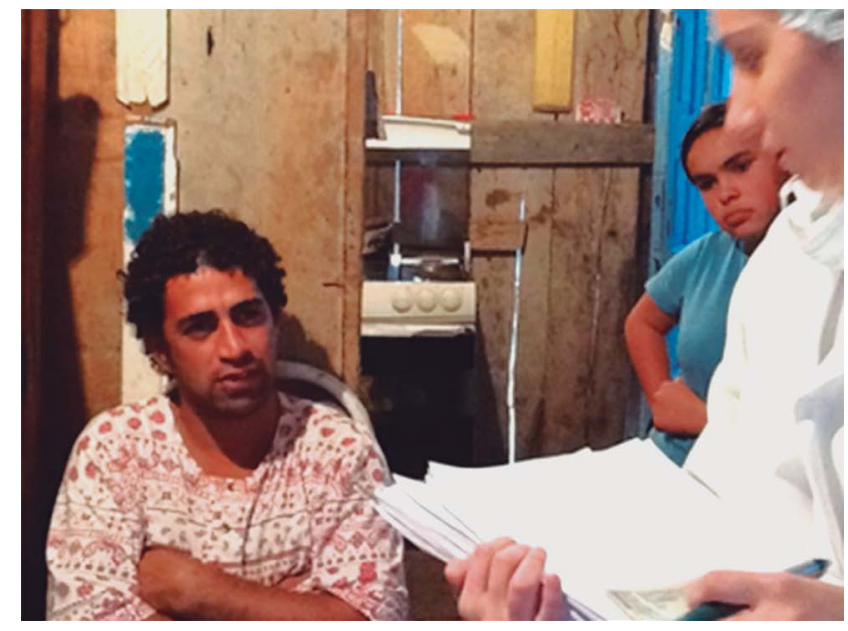

Fonte: dos autores.

Figura 2 - Aplicação do questionário, sendo realizado durante uma visita domiciliar

As condições de saúde bucal desses moradores foram avaliadas utilizando-se os critérios de presença ou de ausência de placa visível e sangramento gengival, número de dentes cariados e perdidos. Esses exames bucais foram realizados por examinadores treinados mediante luz artificial nos ambientes mais claros das residências, sendo iniciados pela verificação da presença de placa visível, seguida da escovação supervisionada e da observação de sangramento gengival. Após essa deplacagem, fez-se a secagem dos dentes com uma gaze e levantou-se o número de dentes cariados, perdidos e restaurados.

Os dados coletados foram armazenados no programa Microsoft Excel 2007 e analisados quantitativamente, com tabelamento em percentual e apresentados em frequência relativa absoluta, de acordo com o teste estatístico Qui-Quadrado $(\mathrm{p}<0,05)$ e, quando houve associação, utilizou-se o resíduo ajustado $\geq 1,96$.

Todos os participantes da pesquisa assinaram um Termo de Consentimento Livre Esclarecido, aprovado pelo Comitê de Ética em Pesquisa da SMS de Porto Alegre sob o número 669, processo n. 001.032690.11.8, datado de 9 de agosto de 2011 .

\section{Resultados}

A Vila Augusta Meneguine, na qual se desenvolveu este estudo, localiza-se no município de Viamão, que é o maior município em extensão territorial da mesorregião Metropolitana de Porto Alegre e da microrregião de Porto Alegre e o sétimo mais populoso do estado do Rio Grande do Sul, com 250 mil habitantes, apresentando em 2010, um Índice de Desenvolvimento Humano Municipal (IDH-M) de 0,717 ${ }^{16}$. 
A amostra desta pesquisa foi constituída de 212 crianças de 0 a 12 anos, pertencentes a aproximadamente 50 famílias, com média de idade de sete anos, sendo $51,4 \%(\mathrm{n}=109)$ do gênero feminino e $48,6 \%$ $(\mathrm{n}=103)$ do gênero masculino.

Em relação ao peso das crianças avaliadas, pode-se constatar que crianças do gênero masculino de três e quatro anos de idade e aquelas a partir dos oito anos tinham peso ligeiramente acima da média estabelecida para crianças brasileiras da mesma idade $^{17}$. Enquanto que para o sexo feminino, esses valores encontram-se acima da média aos três anos e a partir dos cinco anos de idade para essas crianças. Quanto à estatura, crianças do sexo masculino de até $1,5,6,9,10,11$ e 12 anos de idade apresentaram altura abaixo da média. Enquanto que para as idades de até 1 ano, 4, 6 e 12 anos de idade, os valores da estatura mostraram-se ligeiramente abaixo da média, como pode-se analisar claramente nos dados da Tabela 1

Tabela 1 - Comparação da média de peso e altura das crianças avaliadas com relação a média de peso e altura estabelecida para crianças brasileiras da mesma idade. Viamão-RS, 2014.

\begin{tabular}{|c|c|c|c|c|c|c|c|c|c|}
\hline \multirow{3}{*}{ Idade } & \multicolumn{4}{|c|}{ Masculino } & \multirow{3}{*}{ Idade } & \multicolumn{4}{|c|}{ Feminino } \\
\hline & \multicolumn{4}{|c|}{ Média peso /altura (crianças brasileiras) } & & \multicolumn{4}{|c|}{ Média peso/altura (crianças brasileiras) } \\
\hline & Peso (D.E') & Altura (D.E) & Peso (kg) & Altura $(\mathrm{cm})$ & & Peso (D.E) & Altura (D.E) & Peso (kg) & Altura $(\mathrm{cm})$ \\
\hline Até 1 & 10,2400 & $70,00^{*}$ & 11,5 & 81,5 & Até 1 & 9,3886 & $76,71^{*}$ & 10,9 & 81,0 \\
\hline 2 & 12,9945 & 93,73 & 13,9 & 92,0 & 2 & 11,7267 & 94,83 & 13,5 & 90,8 \\
\hline 3 & $18,5333^{*}$ & 104,33 & 16,0 & 98,9 & 3 & $15,7333^{*}$ & 100,17 & 15,4 & 98,3 \\
\hline 4 & $18,4750^{*}$ & $105,40^{*}$ & 18,0 & 106,2 & 4 & 15,3563 & $103,50^{*}$ & 17,6 & 105,3 \\
\hline 5 & 18,6111 & $111,33^{*}$ & 19,9 & 112,0 & 5 & $20,3000^{*}$ & 118,20 & 19,6 & 112,0 \\
\hline 6 & 20,5583 & $115,83^{*}$ & 22,2 & 118,5 & 6 & $22,7000^{*}$ & $117,42^{*}$ & 22,2 & 118,5 \\
\hline 7 & 23,5773 & 125,18 & 25,1 & 124,9 & 7 & $27,5057^{*}$ & 128,43 & 24,9 & 123,3 \\
\hline 8 & $30,8250^{*}$ & 132,50 & 27,7 & 129,7 & 8 & 26,6857 & 132,29 & 27,7 & 129,4 \\
\hline 9 & $37,4650^{*}$ & $134,00^{*}$ & 31,6 & 135,2 & 9 & $33,7423^{*}$ & 137,23 & 31,6 & 135,0 \\
\hline 10 & $35,4750^{*}$ & $138,40^{*}$ & 33,4 & 139,9 & 10 & $40,2167^{*}$ & 141,93 & 33,4 & 140,4 \\
\hline 11 & $41,2000^{*}$ & $142,14^{*}$ & 36,8 & 143,6 & 11 & $42,0000^{*}$ & 148,88 & 36,8 & 147,5 \\
\hline 12 & $43,7444^{*}$ & $149,00^{*}$ & 42,0 & 151,0 & 12 & $42,1900^{*}$ & $152,50^{*}$ & 42,0 & 153,0 \\
\hline
\end{tabular}

Fonte: dos autores.

D.E: Dados estatísticos encontrados neste trabalho

$\left(^{\star}\right)$ : Medidas acima da média estabelecida para crianças brasileiras da mesma idade para o peso e abaixo da média estabelecida para crianças brasileiras da mesma idade para altura.

Diante dos indicadores socioeconômicos, $51 \%$ $(\mathrm{n}=108)$ dos responsáveis disseram ganhar até um salário mínimo por mês. De acordo com o tipo de moradia, $37 \%(\mathrm{n}=79)$ escolheram a opção casa do tipo alvenaria; $29 \%(\mathrm{n}=62)$ de madeira e $29 \%(\mathrm{n}=62)$ disseram ser a construção do tipo mista. Em relação à escolaridade dos responsáveis das crianças que participaram da pesquisa, $30 \%(\mathrm{n}=64)$ disseram ser analfabetos e $62,2 \%(\mathrm{n}=133)$ disseram ter o $1^{\circ}$ grau incompleto. No quesito número de moradores, $51 \%$ $(\mathrm{n}=108)$ responderam morar de três a cinco pes- soas em uma mesma casa e $36 \%(n=77)$ disseram ter mais de cinco moradores. Quanto aos hábitos de saúde bucal, 51,9\% (n=110) responderam que a média de higiene bucal diária era de duas a três vezes ao dia; $35,8 \%(n=76)$ responderam no máximo uma vez ao dia e $12,3 \%(n=26)$ responderam mais de quatro vezes ao dia". Analisando-se os dados de tipo de moradia e a frequência de higiene bucal diária por família, observou-se (Tabela 2) uma correlação estatisticamente significante entre essas variáveis. 
Tabela 2 - Correlação estatisticamente significante entre as variáveis: frequência de higiene bucal diária por família e tipo de casa. Viamão-RS, 2014

\begin{tabular}{|c|c|c|c|c|c|}
\hline \multirow{2}{*}{\multicolumn{2}{|c|}{ Tipo de casa }} & \multicolumn{4}{|c|}{$\begin{array}{l}\text { Frequência de higiene bucal diária } \\
\text { por família }\end{array}$} \\
\hline & & $\begin{array}{l}\text { no máximo } \\
1 \text { vez }\end{array}$ & $\begin{array}{c}\text { de } 2 \text { a } 3 \\
\text { vezes }\end{array}$ & $\begin{array}{c}\text { mais de } 4 \\
\text { vezes }\end{array}$ & total \\
\hline \multirow{3}{*}{ Alvenaria } & $\mathrm{n}$ & 13 & 59 & 7 & 79 \\
\hline & $\%$ & $16,5 \%$ & $74,7 \%$ & $8,9 \%$ & $100,0 \%$ \\
\hline & $\mathrm{RA}^{1}$ & $-4,5^{*}$ & $5,1^{*}$ & $-1,2$ & \\
\hline \multirow{3}{*}{ Madeira } & $n$ & 31 & 22 & 9 & 62 \\
\hline & $\%$ & $50,0 \%$ & $35,5 \%$ & $14,5 \%$ & $100,0 \%$ \\
\hline & $\mathrm{RA}^{1}$ & $2,8^{*}$ & $-3,1^{*}$ & 0,6 & \\
\hline \multirow{3}{*}{ Mista } & $n$ & 29 & 23 & 10 & 62 \\
\hline & $\%$ & $46,8 \%$ & $37,1 \%$ & $16,1 \%$ & $100,0 \%$ \\
\hline & $\mathrm{RA}^{1}$ & $2,1^{*}$ & $-2,8^{*}$ & 1,1 & \\
\hline \multirow{3}{*}{ Outros } & $\mathrm{n}$ & 3 & 6 & 0 & 9 \\
\hline & $\%$ & $33,3 \%$ & $66,7 \%$ & $0 \%$ & $100,0 \%$ \\
\hline & $\mathrm{RA}^{1}$ & $-0,2$ & 0,9 & $-1,1$ & \\
\hline \multirow{2}{*}{ Total } & $n$ & 76 & 110 & 26 & 212 \\
\hline & $\%$ & $35,8 \%$ & $51,9 \%$ & $12,3 \%$ & $100,0 \%$ \\
\hline
\end{tabular}

Fonte: dos autores.

$\left.{ }^{*}\right)$ : RA ${ }^{1}$ - Teste Qui-quadrado de Pearson - Ajuste residual (ponte de corte: $+1,96 ;-1,96)$

Quanto a frequência de ingestão de açúcar entre as refeições (da família), 64,6\% $(\mathrm{n}=137)$ dos cuidadores escolheram a opção três vezes ao dia, enquanto $22,2 \%(\mathrm{n}=47)$ escolheram duas vezes ao dia e $13,2 \%$ $(n=28)$ escolheram uma vez ao dia (Tabela 3$)$.

Tabela 3 - Correlação estatisticamente significante entre renda e frequência de ingestão de açúcar entre as refeições. ViamãoRS, 2014

\begin{tabular}{|c|c|c|c|c|c|}
\hline \multirow{2}{*}{\multicolumn{2}{|c|}{$\begin{array}{l}\text { Renda mensal por } \\
\text { família }\end{array}$}} & \multicolumn{4}{|c|}{$\begin{array}{c}\text { Frequência de ingestão de açúcar } \\
\text { entre as refeições }\end{array}$} \\
\hline & & \multirow{2}{*}{$\begin{array}{l}\begin{array}{l}1 \times \text { ao } \\
\text { dia }\end{array} \\
18\end{array}$} & \multirow{2}{*}{$\begin{array}{r}\begin{array}{c}2 \times \text { ao } \\
\text { dia }\end{array} \\
15\end{array}$} & \multirow{2}{*}{$\begin{array}{r}\begin{array}{c}3 \times \text { ao } \\
\text { dia }\end{array} \\
75\end{array}$} & \multirow{2}{*}{$\begin{array}{r}\text { Total } \\
108\end{array}$} \\
\hline \multirow{3}{*}{$\begin{array}{l}\text { Até } 1 \text { salário } \\
\text { mínimo }\end{array}$} & $\mathrm{n}$ & & & & \\
\hline & $\%$ & $16,7 \%$ & $13,9 \%$ & $69,4 \%$ & $100,0 \%$ \\
\hline & & 1,5 & $-3,0^{*}$ & 1,5 & \\
\hline \multirow{3}{*}{$\begin{array}{l}\text { De } 1 \text { a } 2 \\
\text { salários } \\
\text { mínimos }\end{array}$} & $\mathrm{n}$ & 10 & 23 & 49 & 82 \\
\hline & $\%$ & $12,2 \%$ & $28,0 \%$ & $59,8 \%$ & $100,0 \%$ \\
\hline & & $-0,3$ & 1,6 & $-1,2$ & \\
\hline \multirow{3}{*}{$\begin{array}{l}\text { De } 3 \text { a } 5 \\
\text { salários } \\
\text { mínimos }\end{array}$} & $n$ & 0 & 9 & 13 & 22 \\
\hline & $\%$ & $0 \%$ & $40,9 \%$ & $59,1 \%$ & $100,0 \%$ \\
\hline & $\mathrm{RA}^{1}$ & $-1,9$ & $2,2^{*}$ & $-0,6$ & \\
\hline \multirow{2}{*}{ Total } & & 28 & 47 & 137 & 212 \\
\hline & & $13,2 \%$ & $22,2 \%$ & $64,6 \%$ & $100,0 \%$ \\
\hline
\end{tabular}

Fonte: dos autores.

$\left.{ }^{*}\right):$ RA $^{1}$ - Teste Qui-Quadrado de Pearson - Ajuste residual (ponte de corte: $+1,96 ;-1,96)$

Para a avaliação da pirâmide alimentar foram atribuídos códigos de 0 a 5 para analisarmos quais opções apareciam mais vezes na base da pirâmide, representando quais grupos de alimentos eram mais frequentemente ingeridos, e no topo da pirâmide, representando quais grupos de alimentos eram menos frequentemente ingeridos. $\mathrm{O}$ código 0 foi usado nos casos em que a criança ainda fazia somente uso de mamadeira e/ou do peito. $\mathrm{O}$ código 1 refere-se ao grupo de "oléos e gorduras/açúcares e doces", o código 2 refere-se ao grupo de leite e derivados/ carnes e ovos/leguminosas, o código 3 refere-se ao grupo de hortaliças e frutas e o código 4 refere-se ao grupo de cereais, tubérculos, pães e raízes. A alternativa que mais vezes apareceu na base da pirâmide foi a opção 4, representando $38,2 \%(n=81)$ das respostas, seguido pela opção 2 , representando $28,3 \%(\mathrm{n}=60)$ das respostas.

$\mathrm{Na}$ verificação das condições de saúde bucal das crianças avaliadas, elas foram divididas em grupos de até 3 anos, de 4-6 anos e de 7-12 anos, para facilitar a interpretação dos dados ${ }^{18}$, sendo que o maior número de crianças encontrava-se no grupo de 7-12 $\operatorname{anos}(n=119, f=60, m=59)$. Os resultados são apresentados na Tabela 4.

Tabela 4 - Relação do número de crianças que apresentavam pre-
sença de placa visível, presença de sangramento gengival
à escovação e pelo menos um dente cariado, perdido ou
obturado, divido por faixa etária. Viamão-RS, 2014
Fonte: dos autores.

\section{Discussão}

O município de Viamão apresentou, em 2010, um IDH-M ${ }^{16}$ médio e, considerando esses valores torna-se possível avaliar o quadro de desenvolvimento de um determinado município. Segundo Roncalli $^{19}$ (2008), observa-se uma forte correlação entre o IDH-M e os principais indicadores de saúde, sendo que essa correlação não é recente, tendo em vista que a atenção odontológica no Brasil historicamente é marcada pelas condições socioeconômicas da população. Os três pilares que constituem o IDH-M (saúde, educação e renda) são mensurados por meio da expectativa de vida (saúde); do grau de escolaridade (educação); e do PIB per capita expresso em poder de paridade de compra (renda).

Desse modo, especificamente a amostra deste trabalho foi caracterizada por viver abaixo da linha da pobreza e/ou em extrema pobreza, uma vez que a maioria das famílias relatou renda de até um salário mínimo por mês e, em sua maioria, eram compostas por 3 a 5 moradores por residência, em situação precária. Além disso, o grau de escolaridade 
encontrado foi muito baixo, pois aqueles que escolheram a opção "analfabetos", somados aos que tinham apenas o $1^{\circ}$ Grau Incompleto, representavam mais de $90 \%$ da população descrita. Esses dados exemplificam o perfil socioeconômico vulnerável da amostra, confirmando o motivo pelo qual a maioria dessas famílias sobrevivia basicamente com créditos concedidos pelo governo federal, como o "Programa Bolsa Família”, e, por isso, quando questionados sobre quais alimentos eram mais frequentemente ingeridos, a opção que mais vezes apareceu como respostas referiu-se ao grupo de "cereais, tubérculos, pães e raízes”, itens principais da cesta básica brasileira, pois garantem de $45 \%$ a $65 \%$ da energia total diária da alimentação ${ }^{20}$. Esse resultado vai ao encontro com a análise de consumo alimentar do brasileiro, que constatou que além do arroz e do feijão, as classes de renda mais baixas consomem em maior quantidade vários itens considerados parte de uma dieta saudável, como a farinha de mandioca que é ingerida mais de dez vezes mais na classe de menor renda quando comparada à maior, isso, ocorrendo em relação ao milho, cujo consumo é três vezes maior quando se comparam às classes de menor e de maior renda ${ }^{17}$.

Ainda, associando renda e padrão alimentar, apesar de todos os grupos de renda salarial ter elevado consumo de açúcar entre as refeições, neste estudo, as famílias com renda salarial mais elevada tiveram um maior consumo de açúcar entre as refeições, comparados àquelas com menor renda, cuja frequência de ingestão de açúcar era significativamente menor (Tabela 2). Para Levy et al. ${ }^{21}$ (2012), açúcares e refrigerantes apresentaram padrões mais complexos de consumo, havendo diminuição do consumo de açúcar de mesa e aumento do consumo de refrigerantes conforme a elevação da renda, o que vai ao encontro do resultado obtido nesta pesquisa.

O presente estudo também revelou uma associação significante entre a estrutura da residência (alvenaria, madeira ou mista) e a frequência de higiene bucal por dia realizada pelos moradores, corroborando os achados de Figueiredo et al ${ }^{22}$ (2014), que também verificaram que famílias que residiam em casas mais estruturadas, de alvenaria, apresentaram uma maior frequência de higiene bucal do que aquelas cujas moradias eram feitas de madeira ou mista. Conforme Moreira et al ${ }^{23}$ (2007), deve-se reconhecer uma reprodução de expressão espacial das condições de vida e de saúde da população, na medida em que o local de moradia do indivíduo reflete à sua própria condição socioeconômica.

Por outro lado, considerando o aumento da prevalência da obesidade infantil tanto em países desenvolvidos quanto em países em desenvolvimento como o Brasil $^{24}$, a relação entre cárie na infância e estado nutricional tem sido alvo de recentes estudos, os quais mostram resultados conflitantes. Vários estudos apontaram uma associação entre cárie na infância e déficit antropométricos na altura ${ }^{25}$, no índice de massa corporal ou no peso ${ }^{26}$. Já outros autores encontraram uma associação com a obesidade $^{27}$ e outros não conseguiram estabelecer nenhuma correlação entre cárie em crianças e seu estado nutricional $^{28}$.

O consumo de sacarose foi relatado por Peres et $a .^{25}$ (2005) e Oliveira et al. ${ }^{26}$ (2008) como uma associação positiva no aparecimento da cárie em crianças, embora a ingestão de carboidratos pareça ter uma pequena influência na obesidade ou no sobrepeso dessas ${ }^{29}$. Os achados deste estudo corroboram com tais autore ${ }^{25,26}$, os quais afirmaram existir uma associação positiva entre o consumo de sacarose com o aparecimento da cárie em crianças, uma vez que, a frequência da ingestão de sacarose entre as refeições por $60 \%$ das famílias avaliadas era de três vezes ao dia e mais de $60 \%$ das crianças apresentaram lesões de cárie. Seguindo a mesma lógica de Skinner, et al. ${ }^{29}$ (2004), a ingestão de sacarose pareceu ter uma pequena influência na obesidade ou no sobrepeso dessas, uma vez que em muitas faixas etárias foram observadas crianças com peso e estatura ligeiramente acima da média estabelecida para crianças brasileiras da mesma idade.

Por outro lado, os resultados que demonstraram que, em todas as idades estudadas, os componentes cariados, a presença de placa e o sangramento gengival foram muito prevalentes revelam uma deficiente cobertura na atenção odontológica para as crianças de 0 a 12 anos de idade ou, uma menor importância dada à manutenção da dentição decídua e mista. São as crianças que acumulam grande parte da doença cárie e periodontal, apontando a necessidade de investimento em estratégias de promoção da saúde, incluindo a continuidade das medidas populacionais adotadas nos municípios e a ampliação das medidas educativas e preventivas voltadas a esse grupo populacional, além da ampliação do acesso ao tratamento odontológico de toda a população $0^{30}$.

Pelo mesmo caminho, o componente obturado apareceu em maior percentual na idade de 7 a 12 anos, o que pode caracterizar que as crianças dessa idade tem um maior acesso aos serviços de saúde, porém indica que sejam revistas as ações coletivas de educação em saúde e de prevenção da cárie dentária. Com base na avaliação desses resultados, seria possível planejar ações para a promoção da saúde bucal, que está inserida num conceito amplo de saúde que transcende a dimensão meramente técnica do setor odontológico, promovendo uma integração às demais práticas de saúde coletiva. A construção de políticas públicas saudáveis no município de Viamão significa o desenvolvimento de estratégias direcionadas a todas as crianças, assegurando-lhes a disponibilidade de cuidados odontológicos apropriados.

Finalizando, o envolvimento das crianças, pais e responsáveis, bem como de profissionais da saúde nas atividades é indicado como importante estraté- 
gia que pode oportunizar o reconhecimento de problemas, seus determinantes e fatores de risco associados (padrão alimentar inadequado), favorecendo o empoderamento individual e coletivo. Esse envolvimento também reforça a importância da participação de todos no cuidado com a saúde bucal. Além disso, podem ser importantes momentos de mobilização coletiva para práticas educativas que favoreçam o aprendizado e o controle social sobre as estratégias necessárias neste ambiente comunitário.

\section{Conclusão}

Por meio desse levantamento foi possível identificar os seguintes aspectos:

- uma população com um padrão alimentar caracterizado pela maior ingestão de alimentos à base de carboidratos e um perfil carente de atenção à saúde bucal e de nível socioeconômico com baixa remuneração e escolaridade;

- uma associação positiva existe entre o consumo de sacarose com o aparecimento da cárie em crianças;

- as crianças avaliadas apresentaram altos índices de dentes cariados, perdidos e obturados, placa visível e sangramento gengival;

- uma associação significante foi encontrada entre as variáveis de renda e frequência de mais ingestões de açúcar entre as refeições, nas quais as famílias com renda salarial mais elevada tiveram um maior consumo de açúcar entre as refeições comparadas àquelas com menor renda, cuja frequência de ingestão de açúcar foi significativamente menor; e entre as variáveis "tipo de casa" e "frequência de higiene bucal realizada", a qual era feita com maior frequência no dia pelos moradores de casas do tipo alvenaria.

\section{Agradecimentos}

Os autores agradecem a colaboração da Secretaria de Saúde do Município de Viamão, RS, à coordenadora de Saúde Bucal do referido município, à CD Virgínia Espina e à cirurgiã-dentista da Unidade Básica de Saúde Augusta Meneguini, Dilene Dumke, pelo apoio dado durante a realização desta pesquisa.

\section{Abstract}

Introduction: to provide effective actions in pursuit of oral health, access to health care should be singled out according to the needs of the population, it must be planned from surveys and studies focusing on the profile of the community, and serving as a framework for planning future actions. Objectives: to describe oral health conditions and the dietary pattern of children, and correlate them with the socioeconomic profile of families enrolled in the Family Health Strategy of Augusta Meneguine in the city of Viamão, RS, Brazil. Methodology: this is an observational longitudinal study, in which data were collected from 212 children, from 0 to 12 years of age, and their families during home visits. Results: $42 \%$ of people were living with up to 1 minimum wage, and $48.6 \%$ of households included 3-5 people. There was a significant association between family income of up to 1 minimum wage and more than 3 intakes of sugar in between meals, 3-5 minimum wages and 2 intakes of sugar in between meals; house type (brickwork) and 2 to 3 times a day of oral hygiene $(p<0.05)$. Children showed more than $60 \%$ of decayed teeth, $10 \%$ of missing teeth, and $40 \%$ of filled teeth, in the range of 7-12 years old. Conclusion: through this survey it was possible to identify a poor oral health condition and socioeconomic status of the population studied: low wages and schooling of children's caregivers, high level of visible plaque, gingival bleeding, tooth decay, tooth loss of children.

Keywords: Children. Epidemiology. Dietary pattern. Oral health.

\section{Referências}

1. Wiest JM, Carvalho HH, Figueredo MB, Oliveira MB. Um olhar sobre alimentação, cultura e sociedade. In: Wiest et al. (Ed.). Alimentos e alimentação enquanto saberes e fazeres no Limoeiro do Bacuparí. Porto Alegre: Editora Evangraf; 2008; p. 25-49.

2. Filho MB, Batista LV. Transição alimentar/nutricional ou mutação antropológica. Rev Soc Bras Progresso Ciênc. 2010; 62(4):26-30.

3. United States Department of Agriculture. My pyramid. USA, 1992; Atualizada em 28 set 2010. [Citado 2013 nov]. Disponível em URL: http://www.mypyramid.gov/.

4. Milano MM. Príncipe Kincas e a Grande Pirâmide dos Alimentos. Porto Alegre. Editora Razão; 2006. 46p.

5. Tucunduva PS, Romero LA, Rodrigues CAT, Cisotto RL. Pirâmide alimentar adaptada: guia para escolha dos alimentos. Rev Nutr [serial on the internet]. 1999;12(1):65-80.

6. Batista LRV, Moreira EAM, Corso ACT. Alimentação, estado nutricional e condição bucal da criança Campinas: Pontifícia Universidade Católica de Campinas, 2007.

7. Auad SM, Pordeus IA. Nutrição e sua influência nos processos de odontogênese, erupção e desenvolvimento da cárie dentária. Rev CRO MG. 1999; 5(3):151-5.

8. American Dietetic Association. Position of the American Dietetic Association: oral health and nutrition. J Am Diet Assoc. 1996; 96(2):184-9.

9. Pinto VG. Relacionamento entre padrões de doença e serviços de atenção odontológica. São Paulo: Rev. Saúde Pública 1989, 23:509-14. 
10. Ministério da Saúde. Secretaria de Atenção Básica. Departamento de Atenção Básica. Projeto SB Brasil 2010-Pesquisa Nacional de Saúde Bucal: Resultados principais. Brasília: Ministério da Saúde, 2011.

11. Loretto NRM, Seixas ZA, Jardim MC, Brito R L. Cárie dentária no Brasil: alguns aspectos sociais, políticos e econômicos. Rev da ABO Nacional. 2000; 8:45-9.

12. Monteiro CA, Mondini L, Costa RBL. Mudanças na composição e adequação nutricional da dieta familiar em áreas metropolitanas do Brasil (1988-1996). Rev Saúde Pública 2000; 34(3): 251-8.

13. Furlani PA. Influências dos aspectos sociais e culturais na prevalência da cárie dentária em escolares de Jaraguá do Sul. Monografia (Especialização em Odontologia Preventiva e Social), Curitiba: Universidade Federal do Paraná, 1993.

14. Baldani MH, Narvai PC, Antunes JLF. Cárie dentária e condições sócio-econômicas no Estado do Paraná, Brasil, 1996. Cad. Saúde Pública 2002, 18(3):755-63.

15. Organização Mundial da Saúde. Levantamento epidemiológico em saúde bucal: manual de instruções. Genebra; 1997.

16. Instituto Brasileiro de Geografia e Estatística. Índice de Desenvolvimento Humano Municipal. [Citado 2014 maio 29]. Disponível em URL: <http://cod.ibge.gov.br/16QH>. .

17. Instituto Brasileiro de Geografia e Estatística, Coordenação de Trabalho e Rendimento. Pesquisa de orçamentos familiares 2008-2009: análise do consumo alimentar pessoal no Brasil. Rio de Janeiro: IBGE, 2011.

18. Papália D. et al. Desenvolvimento humano. 8. ed. Porto Alegre: Artmed; 2006.

19. Roncalli AG. Epidemiologia das desigualdades em saúde bucal com foco em famílias. In: Moysés ST, Kriger L, Moysés SJ. Saúde bucal das famílias: trabalhando com evidências. São Paulo: Artes médicas, 2008. p. 170-92.

20. Ministério da Saúde, Secretaria de Atenção à Saúde. Guia alimentar para a população brasileira: promovendo a alimentação saudável - Brasília: Ministério da Saúde; 2008. $210 \mathrm{p}$.

21. Levy RB. et al. Disponibilidade de "açúcares de adição" no Brasil: distribuição, fontes alimentares e tendência temporal. Rev Bras Epidemiol 2012; 15(1):3-12.

22. Figueiredo MC, Peixoto LT, Covatti, F, Silva, KVCL, Jardim LE. Saúde bucal de pessoas em situação de pobreza extrema residentes em um município no sul do brasil. Unopar. Cient Ciênc Biol Saúde 2014;16(1):45-50.

23. Moreira SR, Nico SL, Tomita NE. A relação entre o espaço e a saúde bucal coletiva: por uma epidemiologia georreferenciada. Ciênc Saúde Coletiva 2007;12(1):275-84.

24. Wang Y, Monteiro C, Popkin BM. Trends of obesity and underweight in older children and adolescents in the United States, Brazil, China, and Russia. Am J Clin Nutr. 2002;75(6):971-7.

25. Peres MA, et al. Social and biological early life influenceson severity of dental caries in children aged 6 years. Community Dent Oral Epidemiol. 2005; 33(1):53-63.

26. Oliveira LB, Sheiham A, Bonecker M. Exploring the association of dental caries with social factors and nutritional status in Brazilian preschool children. Eur. J. Oral Sci. 2008;116:37-43.

27. Gerdin EW, Angbratt M, Aronsson K, Eriksson E, Johansson I. Dental caries and body mass index by socio-economic status in Swedish children. Community Dent Oral Epidemiol. 2008; 36(5):459-65.

28. Sheller B, Churchill SS, Williams BJ, Davidson B. Body mass index of children with severe early childhood caries. Pediatri Dent. 2009; 31(3):217-21.
29. Skinner JD, Ziegler P, Pac S, Devaney B. Meal and snack patterns of infants and toddlers. J Am Diet Assoc. 2004; 104(Suppl 1):65-70.

30. Namal N, Yüceokur AA, Can G. Significant caries index values and related factors in 5-6-year-old children in Istanbul, Turkey. East Mediterr Health J. 2009; 15(1): 178-84.

\section{AGENCIADE FOMENTO: Edital PROEXT(SESU/M)}

\section{Endereço para correspondência:}

Márcia Cançado Figueiredo

Universidade Federal do Rio Grande do Sul

(UFRGS)

Faculdade de Odontologia

Ramiro Barcelos, n²492 - Bairro Bom Fim

90035-003 Porto Alegre-RS

E-mail:mcf1958@gmail.com

Recebido: 29/07/2014. Aceito: 05/12/2014. 